\title{
Long-term survival of 11 years with multidisciplinary therapy for hepatocellular carcinoma metastasis to the ovary and peritoneum: a case report
}

\author{
Satoko Motegi ${ }^{1} \cdot$ Takeshi Yokoo $^{1,2}$ (1) $\cdot$ Ryosuke Nozawa $^{1} \cdot$ Rie Azumi $^{1} \cdot$ Yuzo Kawata ${ }^{1} \cdot$ Kohei Ogawa $^{1} \cdot$ Toru Setsu $^{1}$. \\ Ken-ichi Mizuno ${ }^{1} \cdot K_{\text {Koji Nishino }}^{3} \cdot$ Hajime Umezu $^{4} \cdot$ Hirokazu Kawai $^{5} \cdot$ Takeshi Suda $^{6} \cdot$ Shuji Terai $^{1}$
}

Received: 4 February 2021 / Accepted: 2 May 2021 / Published online: 12 May 2021

(c) The Author(s) 2021

\begin{abstract}
We herein report a rare case of HCC metastases to the ovary and peritoneum in a 61-year-old female patient who has achieved 11-year survival with multidisciplinary therapy. The patient was diagnosed with HCC during balloon angioplasty performed for Budd-Chiari syndrome in 1994 and underwent partial hepatectomy twice. Five years after the second hepatectomy, allochronic recurrence of a single nodule detected in $\mathrm{S} 8$ was treated by radiofrequency ablation, followed by percutaneous ethanol injection therapy and stereotactic body radiotherapy. However, her $\alpha$-fetoprotein level rose to $1862 \mathrm{ng} / \mathrm{mL}$ within one year and computed tomography revealed a large pelvic tumor suggesting HCC metastasis to the ovary. The subsequent laparotomy revealed one $11-\mathrm{cm}$ left ovarian tumor, one small right ovarian nodule, and numerous peritoneal nodules. Bilateral salpingo-oophorectomy and peritoneal resection of as many nodules as possible were performed. Combination therapy with intravenous 5-fluorouracil plus cisplatin and ramucirumab monotherapy effectively suppressed tumor progression with maintenance of hepatic functional reserve, and she has achieved long-term survival of 11 years, illustrating that multidisciplinary therapy with favorable hepatic functional reserve maintenance can contribute to long-term survival in HCC with extrahepatic spread.
\end{abstract}

Keywords Hepatocellular carcinoma $\cdot$ Ovary $\cdot$ Peritoneum $\cdot$ Multidisciplinary therapy $\cdot$ Case report

Takeshi Yokoo

t-yokoo@med.niigata-u.ac.jp

1 Division of Gastroenterology and Hepatology, Graduate School of Medical and Dental Sciences, Niigata University, Niigata, Niigata, Japan

2 Department of Preemptive Medicine for Digestive Diseases and Healthy Active Life, School of Medicine, Niigata University, Niigata, Niigata, Japan

3 Department of Obstetrics and Gynecology, Graduate School of Medical and Dental Sciences, Niigata University, Niigata, Niigata, Japan

4 Division of Pathology, Graduate School of Medical and Dental Sciences, Niigata University, Niigata, Niigata, Japan

5 Department of Internal Medicine, Niigata Prefectural Shibata Hospital, Shibata, Niigata, Japan

6 Department of Gastroenterology and Hepatology, Uonuma Institute of Community Medicine Niigata University Hospital, Minamiuonuma, Niigata, Japan

\section{Introduction}

The lungs, lymph nodes, bones, and adrenal glands are the most common metastatic sites, and the reported median survival after the diagnosis of extrahepatic spread is 8.1 months [1]. Importantly, the prognosis remains poor in these patients despite the recently developed molecular-targeted agents. We herein report the case of a patient with rare ovarian and peritoneal metastases of HCC who achieved 11-year survival by longitudinal multidisciplinary therapy, which afforded effective maintenance of hepatic functional reserve.

\section{Case report}

A 61-year-old female patient was diagnosed with liver cirrhosis due to Budd-Chiari syndrome and underwent balloon angioplasty in 1994. At the time, she was also diagnosed with HCC located in S7 and underwent partial hepatectomy one month later. Eight years after the 
initial HCC diagnosis, two new HCC nodules found in S2 and $\mathrm{S} 3$ were removed by partial hepatectomy as curative surgery. Five years after the second hepatectomy, she experienced another HCC recurrence in S8, which presented as corona-like enhancement in computed tomography (CT) images during hepatic arteriography (Fig. 1). Although sequential treatment with radiofrequency ablation (RFA) and percutaneous ethanol injection therapy (PEIT) was performed, local recurrence was detected seven months after the treatment. The patient underwent RFA again. As a result, a liver abscess was developed at the ablation site. After the resolution of abscess with antibiotic treatment, remnant HCC was visualized. Stereotactic body radiotherapy was performed as additional treatment eleven months after the second RFA.

However, her $\alpha$-fetoprotein (AFP) level reached $1862 \mathrm{ng} / \mathrm{mL}$ ten months after stereotactic body radiotherapy (about 2.5 years later from diagnosis of S8 nodule). Neither CT nor magnetic resonance imaging (MRI) showed HCC recurrence in the liver or the upper abdomen, but gynecological examination performed to diagnose lower abdominal discomfort led to the identification of a non-tender, hard mass in the lower abdomen.

The results of laboratory tests performed at the time are presented in Table 1. The levels of AFP and des- $\gamma$ carboxy prothrombin (DCP) were elevated $(2156 \mathrm{ng} / \mathrm{mL}$ and $2302 \mathrm{mAU} / \mathrm{mL}$, respectively), whereas CA125 was slightly above the normal limit $(123 \mathrm{U} / \mathrm{mL})$. Her hepatic functional reserve was Child-Pugh A (6 points), and she was in modified albumin-bilirubin (mALBI) grade $2 \mathrm{a}$ (score, -2.45 ). Her renal function was not impaired. MRI indicated a large tumor measuring $11 \times 10 \times 6 \mathrm{~cm}$ in
Table 1 Laboratory findings at the time of ovarian tumor diagnosis

\begin{tabular}{llllll}
\hline TP & 7.9 & $\mathrm{~g} / \mathrm{dL}$ & AFP & 2156 & $\mathrm{ng} / \mathrm{mL}$ \\
Alb & 3.8 & $\mathrm{~g} / \mathrm{dL}$ & AFP-L1 & 95.3 & $\%$ \\
T-Bil & 0.9 & $\mathrm{mg} / \mathrm{dL}$ & AFP-L3 & 4.7 & $\%$ \\
AST & 50 & $\mathrm{U} / \mathrm{L}$ & DCP & 2302 & $\mathrm{mAU} / \mathrm{mL}$ \\
ALT & 22 & $\mathrm{U} / \mathrm{L}$ & CA125 & 123 & $\mathrm{U} / \mathrm{mL}$ \\
BUN & 20 & $\mathrm{mg} / \mathrm{dL}$ & CEA & 5.1 & $\mathrm{ng} / \mathrm{mL}$ \\
Cre & 0.54 & $\mathrm{mg} / \mathrm{dL}$ & CA19-9 & 17 & $\mathrm{U} / \mathrm{mL}$ \\
NH3 & 113 & $\mu \mathrm{g} / \mathrm{dL}$ & & & \\
PLT & 19.6 & $\times 10^{4} / \mathrm{uL}$ & Encephalopathy & & None \\
PT & 85 & $\%$ & Ascites & & Mild \\
PT-INR & 1.09 & & & & \\
& & & Child-Pugh & A (6) \\
& & & mALBI grade & $2 \mathrm{a}(-2.45)$ \\
& & & FIB-4 index & 3.32 \\
\hline
\end{tabular}

the pelvic cavity (Fig. 2a-d), and the ovary was considered as the origin of the tumor, which was hypervascular and included necrotic areas. No HCC recurrence was detected in the liver by dynamic CT and MRI. No tumor was observed in lymph nodes, lungs, bones, and adrenal glands. Endoscopy did not show any tumors in esophagus, stomach, duodenum, and colon. The pelvic tumor was strongly suspected to be an HCC metastasis based on the patient's medical history, high levels of AFP and DCP, and no evidence of tumors in other organs.

Bilateral salpingo-oophorectomy as curative therapy was attempted in December 2009. However, in addition to the large ovarian tumor, numerous small nodules in the peritoneum and mesentery were detected
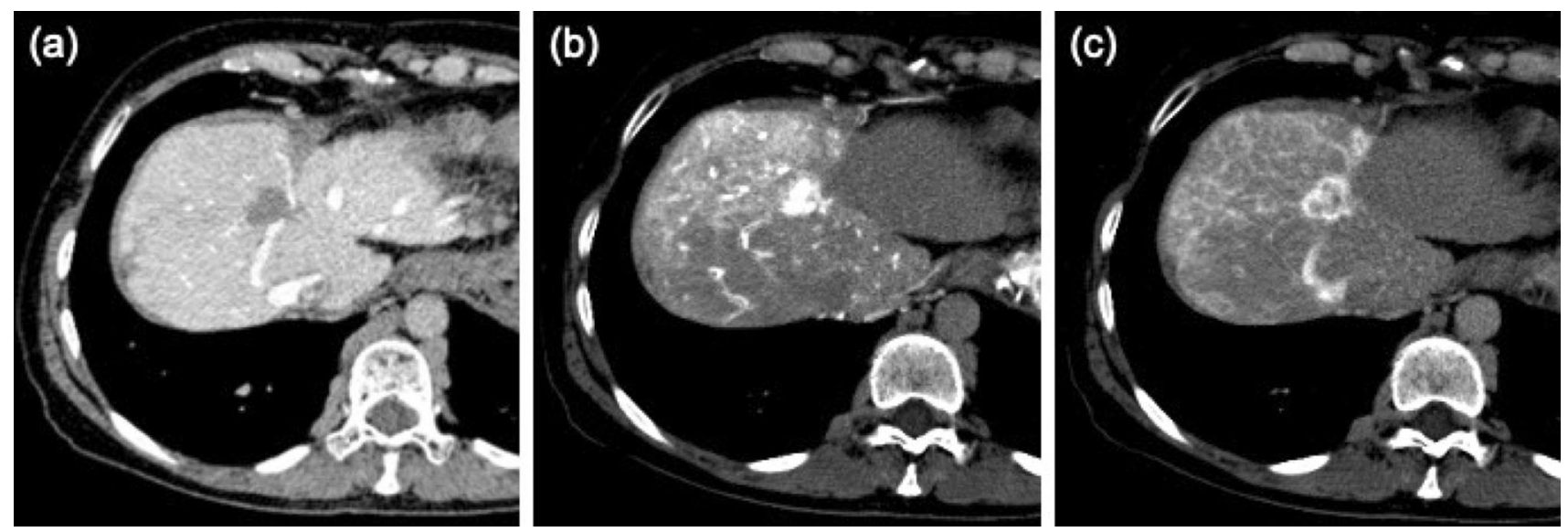

Fig. 1 Computed tomography images during angiography at the time of S8 nodule diagnosis in 2007. a Computed tomography (CT) during arterioportography image showing a defect in the portal supply to the tumor. b, c CT during right hepatic arteriography images showing overt enhancement followed by corona-like enhancement, a typical presentation of hepatocellular carcinoma 
Fig. 2 Magnetic resonance images of pelvis. a T2-weighted sagittal image showing a $11-\mathrm{cm}$ tumor including cystic (arrows) and necrotic lesions with hemorrhage (arrowheads). b-d Dynamic contrast-enhanced magnetic resonance images showing tumor hypervascularity (b plain phase; c early phase; $\mathbf{d}$ delayed phase)
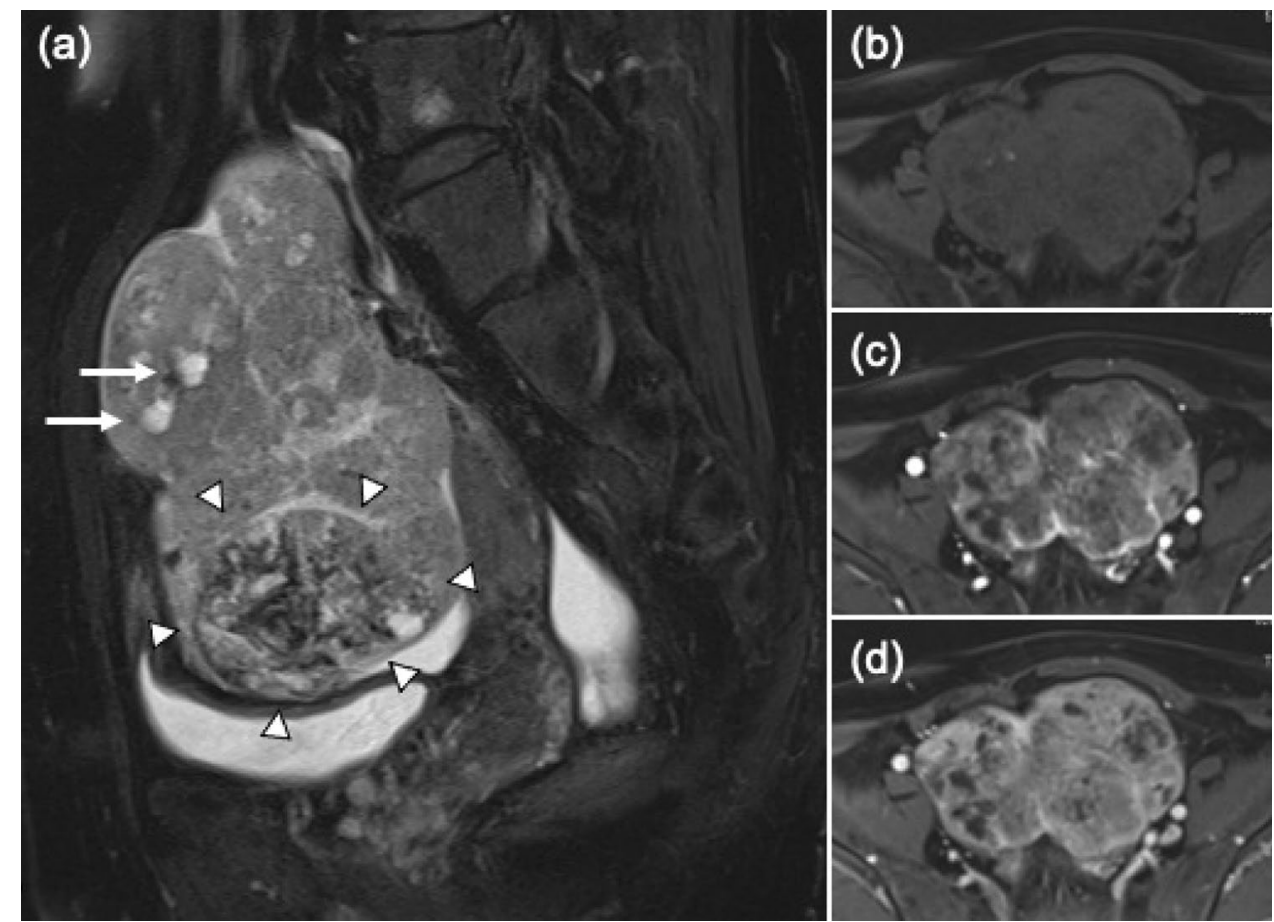

Fig. 3 Gross findings of the pelvic cavity during laparotomy. a Findings in the pelvic cavity. Left ovary is the origin of the large tumor. Black circles indicate peritoneal nodules. b Gross view of the resected tumor originating from the left ovary. The tumor dimensions are $11 \times 10 \times 6 \mathrm{~cm}$. c The internal structure is yellowish and solid, and partial intertumoral hemorrhage is visible
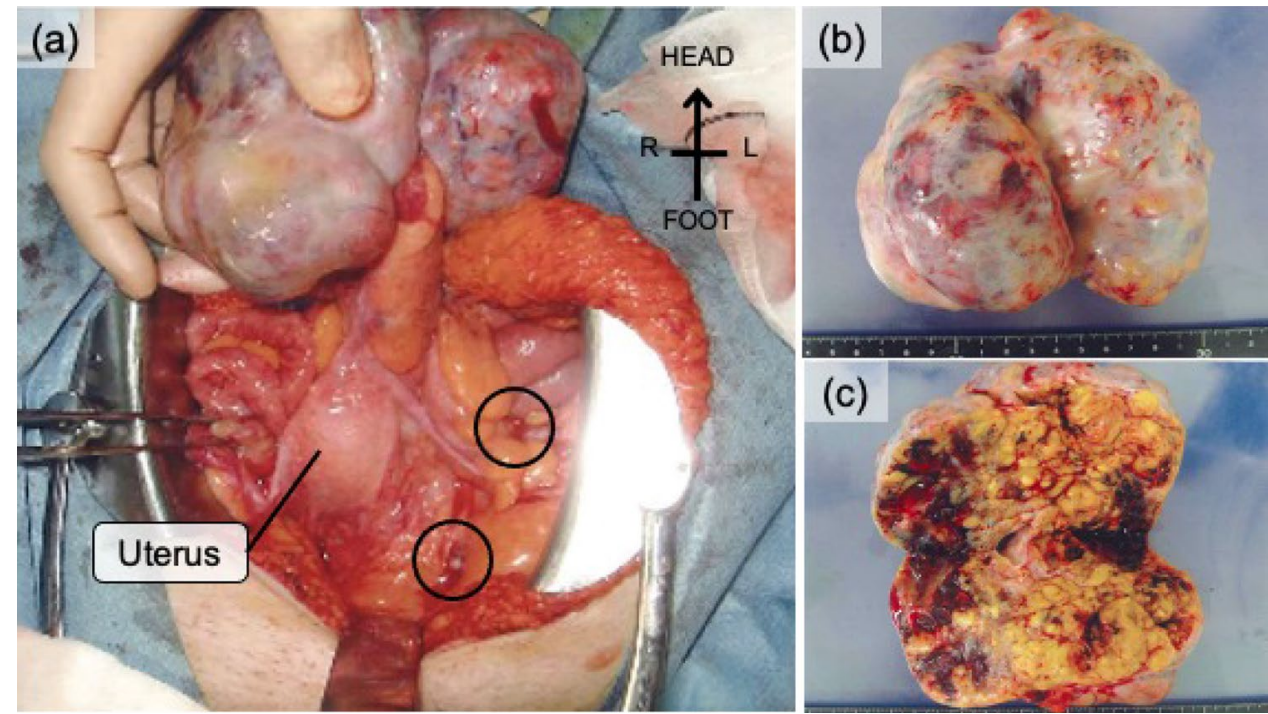

during laparotomy, and as many disseminated nodules as possible were resected in addition to bilateral salpingo-oophorectomy.

Pathological evaluation revealed a yellow solid tumor in the left ovary, $11 \times 10 \times 6 \mathrm{~cm}$ in size, with hemorrhagic and cystic components, partially with aggregations of small nodules (Fig. 3). A nodule, $1.3 \times 0.9 \mathrm{~cm}$ in size, was detected in the right ovary. Microscopic evaluation showed similar findings in both ovarian tumors, which exhibited alveolar, trabecular, and tubular patterns and comprised eosinophilic cells with a high nuclear/cytoplasm ratio. Immunohistochemical examination showed that the tumors were extensively positive for CAM5.2, partially positive for Hep-Par1 (Fig. 4c) and CK7 (Fig. 4d), and negative for CK20 and AE1/AE3 (data 

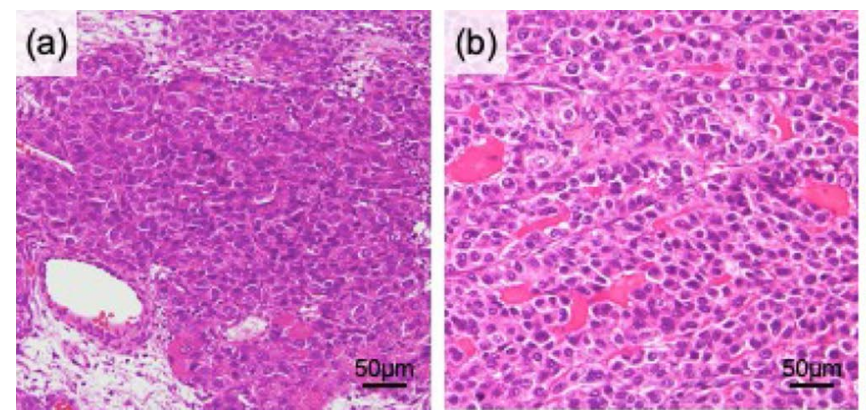

Fig. 4 Representative microscopic images of the left ovarian tumor. a, b Hematoxylin-eosin staining. Eosinophilic cells with high nuclear/cytoplasm ratio propagate in a compact pattern. $\mathbf{c}$ Cells posi-
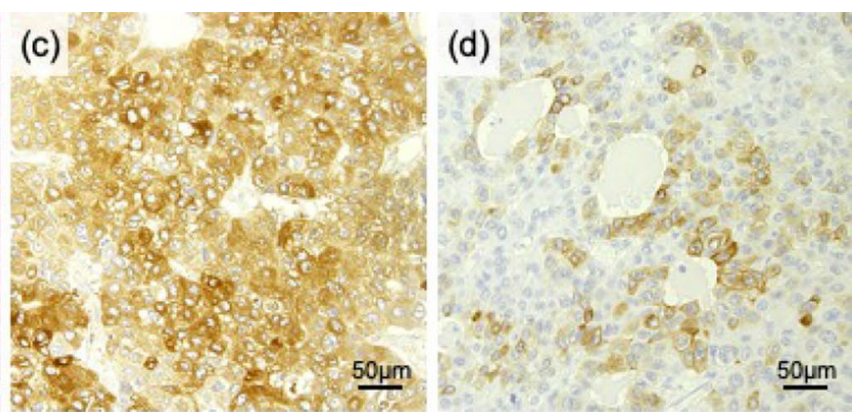

tive for Hep-Par1 are heterogeneously detected in the tumor. d CK7positive cells forming glandular structures are partly observed in the tumor

in common terminology criteria for adverse events version 5.0. Starting in September 2010, 5-fluorouracilbased regimens, such as an intravenous 5-fluorouracil plus cisplatin (low-dose FP; 5-fluorouracil, $250 \mathrm{mg} /$ body, days $1-5,8-12$, and $15-19$ and cisplatin, $10 \mathrm{mg} /$ body, days $1-5,8-12$, and 15-19), and tegafur/gimeracil/ oteracil (S-1, $60 \mathrm{mg} /$ day) monotherapy were administered. Although the regimens suppressed an increase of AFP level under $250 \mathrm{ng} / \mathrm{mL}$ for a year, a new intrahepatic lesion was detected in September 2012. On-demand transarterial chemoembolization (TACE) was added as locoregional therapy. In March 2015, the patient developed anaphylactic reaction to iodine-containing contrast

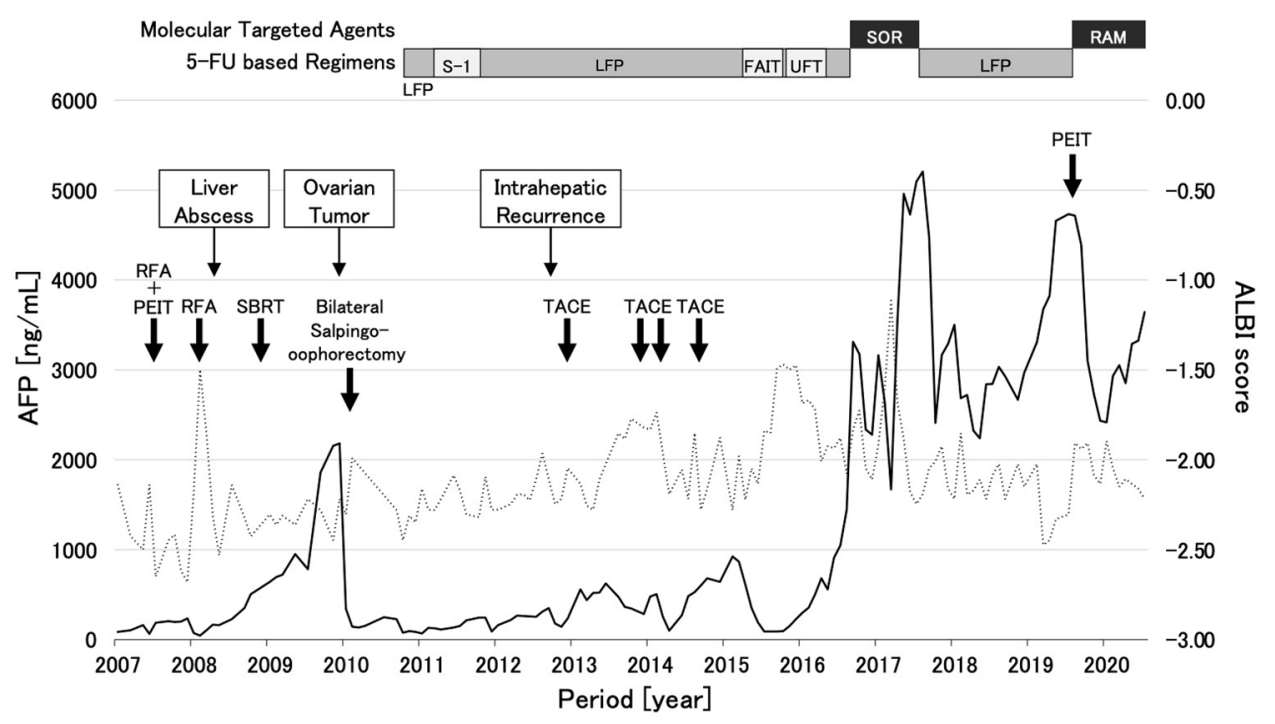

Fig. 5 Clinical course during multidisciplinary therapy. Solid and dotted lines show changes in serum alpha-fetoprotein (AFP) levels (ng/mL) and modified albumin-bilirubin score, respectively, during the course of multidisciplinary therapy. 5-FU 5-fluorouracil, LFP intravenous low-dose 5-FU and cisplatin, $S$-1 tegafur/gimeracil/ oteracil, FAIT Fluorouracil arterial infusion and interferon therapy, $U F T$ tegafur/uracil, SOR sorafenib, $R A M$ ramucirumab, $R F A$ radiofrequency ablation, PEIT percutaneous ethanol injection therapy, $S B R T$ stereotactic body radiation therapy, TACE transarterial chemoembolization 
medium, leading to the contraindication of TACE and contrast-enhanced CT; therefore, the patient was treated with 5-fluorouracil-based regimens, such as intravenous 5 -fluorouracil plus interferon $\alpha$-2a, and tegafur/uracil monotherapy. However, long-term tumor suppression was not possible, and transient reduction in hepatic functional reserve was observed. Renal function also started to decline from this period. Because estimated glomerular filtration rate decreased to $56.05 \mathrm{~mL} / \mathrm{min} / 1.73 \mathrm{~m}^{2}$, low-dose sorafenib ( $200 \mathrm{mg} /$ day) was administered as an alternative treatment and the severity of adverse effects, such as hand-foot syndrome and anorexia, were grade 2 and tolerable. However, the AFP level increased to $5096 \mathrm{ng} / \mathrm{mL}$. Lenvatinib ( $4 \mathrm{mg}$ ), which was initiated as the next treatment, had to be discontinued at day 11 due to a reduction in estimated glomerular filtration rate from 41.01 to $29.31 \mathrm{~mL} / \mathrm{min} / 1.73 \mathrm{~m}^{2}$. After the discontinuation, renal function returned to the former level. The subsequent re-initiation of intravenous low-dose FP with $30 \%$ dose of cisplatin could suppress tumor progression until 2018, as shown in Figs. 5 and 6.

In 2019, four hepatic lesions were newly detected by plain CT and contrast-enhanced ultrasound. Percutaneous ethanol injection therapy for the intrahepatic nodule in S5, which grew more rapidly compared to the other three nodules, was followed by ramucirumab monotherapy. Although the treatment was briefly interrupted due to ophthalmic surgery and proteinuria, her AFP level transiently decreased from 4717 to $2418 \mathrm{ng} / \mathrm{mL}$. With the sequential treatment, the patient has been able to maintain improved quality of life and good performance status for 11 years after the initial diagnosis of HCC metastases to the ovary and peritoneum. Because her mALBI score has exhibited only a slight increase from -2.45 to -2.30 despite the long-term treatment, multidisciplinary treatment has been ongoing.

\section{Discussion}

Ovarian involvement is extremely rare in HCC. To date, only 16 living cases of HCC metastasis to the ovary have been reported except the present case (Table 2) [2-15]. The first two cases were reported in 1983 by Nakashima et al., who reported one case $(0.4 \%)$ in an autopsy cohort of $232 \mathrm{HCC}$ cases [16], and by Oortman et al., who reported a living patient [2]. The median age of these reported cases is 44 (range 27-76) years. Unilateral and bilateral involvement was observed in 9 and 8 patients, respectively, and 8 of the 17 cases had peritoneal metastasis.
In patients with $\mathrm{HCC}$ metastasis to the ovary, hepatoid yolk sac tumor and primary hepatoid carcinoma of the ovary, both AFP-producing ovarian tumors, should be considered in differential diagnosis. Hepatoid yolk sac tumor is commonly reported in young female patients, with rare occurrence reported in postmenopausal women [17]. Hepatoid carcinoma of the ovary is an uncommon ovarian tumor, first reported in 1987 [18]. The morphological and immunohistochemical features of hepatoid carcinoma of the ovary resemble those of HCC [19-21]. In the present case, the pathological findings were compatible to HCC. Furthermore, the postmenopausal age, history of radiofrequency ablation therapy and percutaneous ethanol injection therapy which can cause ovarian and peritoneal involvement [3,22], and no evidence of tumors in other organs strongly supported the diagnosis of HCC metastasis to the ovary.

Prognosis of HCC with extrahepatic spread is poor. A meta-analysis reported that 1-year survival rate was $25 \%$ in the advanced stage of Barcelona clinic liver cancer staging system [23], and the reported median survival time of extrahepatic spread is approximately 8 months $[1,24]$. Ovarian metastases tend to be detected after they become large and exhibit peritoneal involvement, as shown in Table 2, and regular pelvic examination might be essential in patients with HCC, especially after RFA and PEIT. In the present case, it could be retrospectively assumed that the ovarian metastasis emerged in the duration between the second RFA and stereotactic body radiotherapy, when sufficient investigation of the pelvic cavity was not performed. Continuous increase of AFP after the second RFA might reflect the ovarian metastasis. Actually, AFP reflected progression of peritoneal dissemination and intrahepatic lesions after the salpingooophorectomy. Several studies reported that radical or palliative surgery of extrahepatic sites could significantly prolong survival in patients with good tumor control in the liver and other organs [3, 4, 12, 25]. Some studies described that surgical resection of peritoneal metastasis could also prolong survival in cases with selected conditions, such as small number of peritoneal nodules, AFP level of less than $200 \mathrm{ng} / \mathrm{mL}$, Child-Pugh A, and well-controlled intrahepatic lesion [26-28]. The median survival time of the reported cases of HCC with ovarian metastasis is 12 months (range 4-54), whereas longer survival times beyond 12 months are expected in patients with previously visible $\mathrm{HCC}$ lesions across the body that disappear after surgery. 

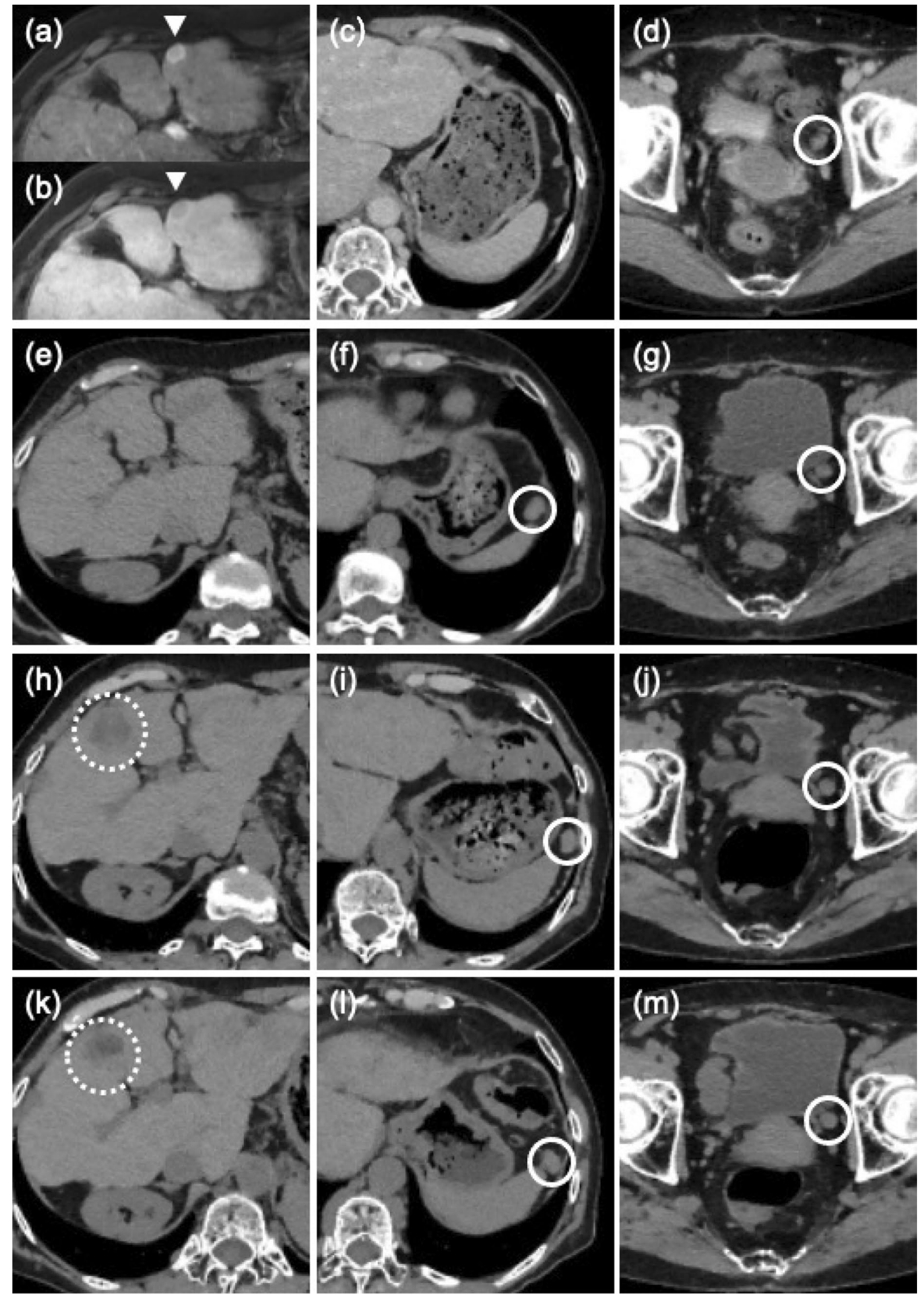
4Fig. 6 Representative images of the liver and abdomen after bilateral salpingo-oophorectomy. Intrahepatic recurrence was detected in 2012. Dynamic MRI showed early enhancement and wash out in early and delayed phase (arrow heads in a, b). Abdominal dissemination was indicated in the pelvis (d) and no nodule was near the spleen (c). Before administration of low-dose sorafenib in 2016 (e, f, g), a new disseminated lesion appeared near the spleen. Use of the contrast medium was avoided due to allergic reaction. Before percutaneous ethanol injection therapy (PEIT) in 2019, another intrahepatic lesion in S5 was clarified even in plain CT (h). The lesions of the abdominal dissemination were stable (i, $\mathbf{j}$ ). After PEIT in 2020, The intrahepatic lesion in S5 include low density area which allowed to suggest necrosis $(\mathbf{k})$. The lesions of the abdominal dissemination were stable $(\mathbf{l}, \mathbf{m})$. White solid and dotted circles show lesions of abdominal dissemination and an intrahepatic lesion in S5

The present case has survived for 11 years after bilateral salpingo-oophorectomy. We propose that three major factors contributed to the prolonged survival with improved quality of life in the present case. First, surgical resection of the large ovarian tumor might have significantly reduced the HCC bulk in the body. Second, effectiveness of 5-fluorouracil-based regimens to peritoneal nodules might have contributed to suppress tumor progression in our case. The remnant lesions after the salpingo-oophorectomy were located at the peritoneum but not in the liver. Targeting the peritoneal nodules, sorafenib was selected as the first treatment, which was the only evidence-based therapy in those days. The SHARP trial reported that sorafenib was effective in prolonging median survival and time to progression in patients with unresectable advanced HCC [29]. Therefore, it was decided that the present case received sorafenib after recovery from surgical wound as the first chemotherapy. Unfortunately, the present case did not have tolerability to sorafenib, and the duration of administration was only 12 days. The 5 -fulorouracil-based regimens were administered from the venous route as an alternative therapy, according to the previous reports [30-32] that described the efficacy of intraarterial 5-fulorouracil-based regimens with cisplatin or interferon $\alpha$. Monotherapy of S-1 was also tried, based on the previous report [33]. Currently, it was known that S-1 did not statistically improve overall survival in patients with at least sorafenib-refractory HCC [34].

Finally, hepatic functional reserve could be maintained during multidisciplinary therapy. The significance of hepatic functional reserve in achieving prolonged survival has been frequently reported in patients undergoing surgical resection [27, 35, 36], locoregional therapy [37-39], and molecular-targeted therapy [40-43]. In the present case, balloon angioplasty performed for
Budd-Chiari syndrome stopped continuous progression of liver fibrosis and prevented the reduction of hepatic functional reserve. In addition, successful suppression of the intrahepatic lesions by 5 -fluorouracil-based regimens aided in minimizing the number of TACE sessions. Low-dose FP was administered approximately three courses per year. As a result, sufficient periods for recovery from adverse effect were obtained and hepatic functional reserve was retained. Ramucirumab also contributed to the suppression of tumor progression without reduction of hepatic functional reserve. In the REACH-2 trial, ramucirumab was associated with improved overall survival compared with placebo [44]. Although the objective response was extremely low, there is a notable case report that continuous ramucirumab treatment for ten months led to partial response with a normal AFP level [45]. Regarding treatment-related adverse effects, good tolerance even in elderly patients was also reported with improvement of overall survival and quality of life [46]. In a network meta-analysis, Wang et al. found that the efficacy of ramucirumab was comparable to regorafenib and cabozantinib in patients with AFP levels above $400 \mathrm{ng} / \mathrm{mL}$, although the adverse effects were fewer with ramucirumab compared to regorafenib and cabozantinib [47]. In the present case, ramucirumab did not decrease tumor volume but dramatically reduced AFP level, and the tolerance was better for ramucirumab than for sorafenib and lenvatinib.

Patients with HCC metastasis to the ovary are usually diagnosed with large-sized tumors with peritoneal involvement; therefore, regular surveillance of the pelvis should be considered especially after hepatic resection, tumor puncture, and HCC rupture. Even in patients with ovarian and peritoneal metastases, multidisciplinary treatment with maintenance of hepatic functional reserve has a possibility of improvement in survival.

In conclusion, multidisciplinary therapy has achieved long-term survival of 11 years with improved quality of life and good performance status in a patient with rare $\mathrm{HCC}$ metastasis to the ovary and peritoneum due to favorable maintenance of hepatic functional reserve. 


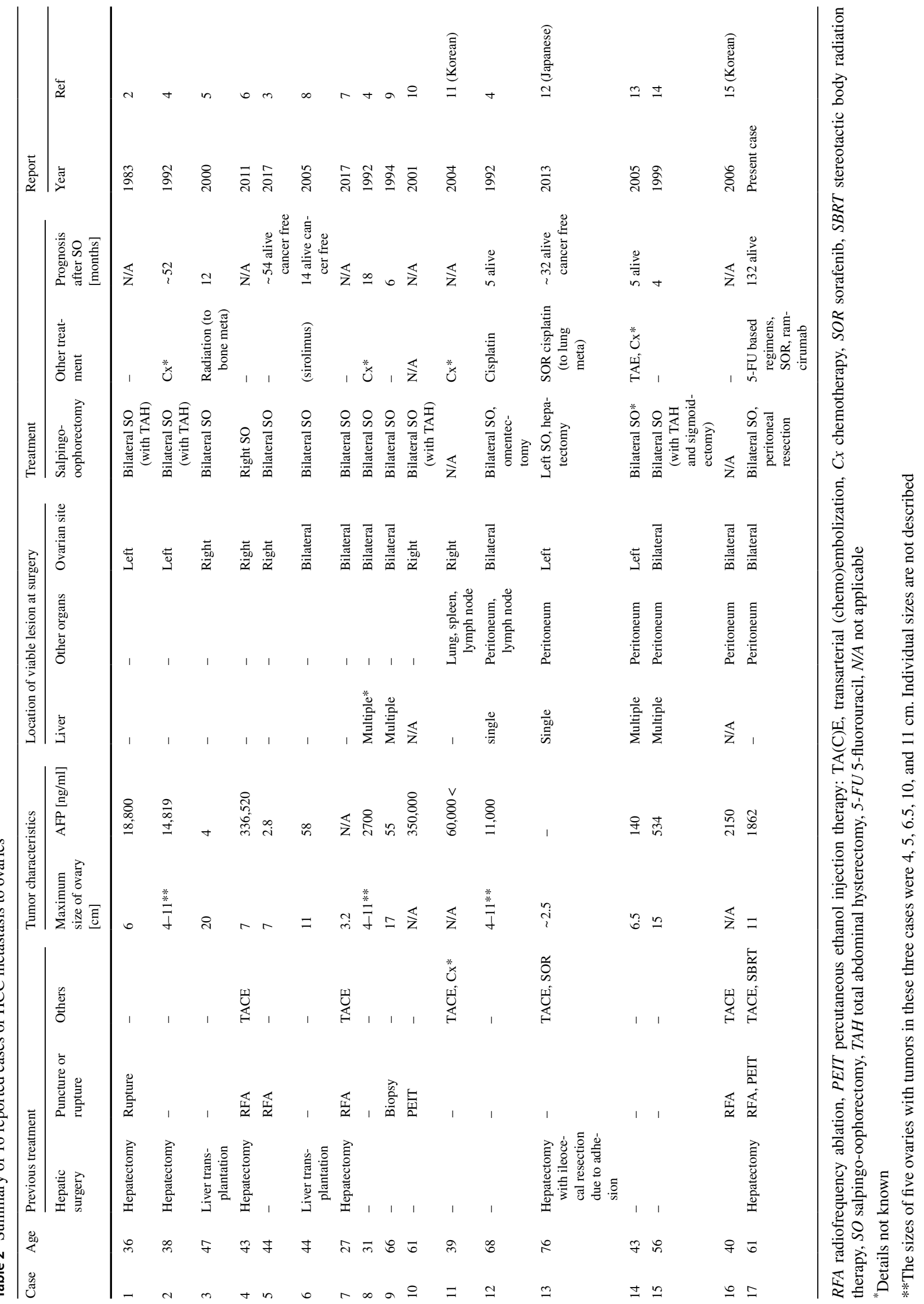


Acknowledgements The authors thank Enago for the critical reading of the manuscript and English language review.

\section{Declarations}

Conflict of interest Satoko Motegi, Takeshi Yokoo, Ryosuke Nozawa, Rie Azumi, Yuzo Kawata, Kohei Ogawa, Toru Setsu, Ken-ichi Mizuno, Koji Nishino, Hajime Umezu, Hirokazu Kawai, Takeshi Suda and Shuji Terai declare that they have no conflict of interest.

Human rights All procedures followed have been performed in accordance with the ethical standards laid down in the 1964 Declaration of Helsinki and its later amendments.

Informed consent Informed consent was obtained from all patients for being included in the study.

Open Access This article is licensed under a Creative Commons Attribution 4.0 International License, which permits use, sharing, adaptation, distribution and reproduction in any medium or format, as long as you give appropriate credit to the original author(s) and the source, provide a link to the Creative Commons licence, and indicate if changes were made. The images or other third party material in this article are included in the article's Creative Commons licence, unless indicated otherwise in a credit line to the material. If material is not included in the article's Creative Commons licence and your intended use is not permitted by statutory regulation or exceeds the permitted use, you will need to obtain permission directly from the copyright holder. To view a copy of this licence, visit http://creativecommons.org/licenses/by/4.0/.

\section{References}

1. Uchino K, Tateishi R, Shiina S, et al. Hepatocellular carcinoma with extrahepatic metastasis: clinical features and prognostic factors. Cancer. 2011;117:4475-83.

2. Oortman EH, Elliot JP. Hepatocellular carcinoma metastatic to the ovary: a case report. Am J Obstet Gynecol. 1983;146:715-7.

3. Yoon SW, Jeong J-S, Kim SY, et al. A Case of needle-tract implantation of hepatocellular carcinoma in the ovary after radiofrequency ablation. Kosin Med J. 2017;32:251.

4. Ishizaki M, Kaibori M, Matsui K, et al. A case of advanced hepatocellular carcinoma in which complete response was achieved with multimodality therapy. Gan To Kagaku Ryohols. 2013;40:1678-80.

5. Kim MJ. A case of metastatic hepatocellular carcinoma of the ovary: an immunohistochemical study and literature review. Korean J Pathol. 2005;39:287-90.

6. Khunamornpong S, Siriaunkgul S, Chunduan A. Metastatic hepatocellular carcinoma of the ovary. Int J Gynecol Obstet. 1999;64:189-91.

7. Lim TK, Uhm JE, Cheong H-S, et al. A case of hepatocellular carcinoma with ovarian metastasis. Korean J Med. 2006;71:573-6.

8. Young RH, Gersell DJ, Clement PB, et al. Hepatocellular carcinoma metastatic to the ovary: a report of three cases discovered during life with discussion of the differential diagnosis of hepatoid tumors of the ovary. Hum Pathol. 1992;23:574-80.

9. De Groot ME, Dukel L, Chadha-Ajwani S, et al. Massive solitary metastasis of hepatocellular carcinoma in the ovary two years after liver transplantation. Eur J Obstet Gynecol Reprod Biol. 2000;90:109-11.
10. Lee JM, Park KM, Lee SY, et al. Metastasis of hepatocellular carcinoma to the ovary: a case report and review of the literature. Gut Liver. 2011;5:543-7.

11. Cil I, Zirtiloglu A, Velibeyoglu MF, et al. What is the optimal treatment of metastatic hepatocellular carcinoma after complete resection; case presentation of ovarian metastasis-PubMed. J BUON. 2017;22:557-8.

12. Stippel DL, Kasper HU, Schleimer K, et al. Successful use of sirolimus in a patient with bulky ovarian metastasis of hepatocellular carcinoma after liver transplantation. Transplant Proc. 2005;37:2185-7.

13. Böcher WO, Löhr HF, Steegmüller KW, et al. Detection of hepatitis $\mathrm{C}$ virus replication in ovarian metastases of a patient with hepatocellular carcinoma. J Hepatol. 1994;21:47-51.

14. Park JH, Han CD, Huh CK, et al. Hepatocellular carcinoma metastatic to the ovary: one case report and review literature. Korean $\mathrm{J}$ Obstet Gynecol. 2001;44:1900-4.

15. Kim TH, Cheung DY, Chung WB, et al. A case of metastatic hepatocellular carcinoma of the ovary. Korean J Gastroenterol. 2004;43:215-8.

16. Nakashima T, Okuda K, Kojiro M, et al. Pathology of hepatocellular carcinoma in Japan: 232 consecutive cases autopsied in ten years. Cancer. 1983;51:863-77.

17. Wang Y, Yang J, Yu M, et al. Ovarian yolk sac tumor in postmenopausal females: a case series and a literature review. Medicine (Baltimore). 2018;97.

18. Ishikura H, Scully RE. Hepatoid carcinoma of the ovary. A newly described tumor. Cancer. 1987;60:2775-84.

19. Pandey M, Truica C. Hepatoid carcinoma of the ovary. J Clin Oncol. 2011;29.

20. Wang L, Zhong Y, Sun L, et al. Clinical and pathological features of hepatoid carcinoma of the ovary. World J Surg Oncol. 2013;11.

21. Choi WK, Cho DH, Yim CY, et al. Primary hepatoid carcinoma of the ovary: a case report and review of the literature. Medicine (Baltimore). 2020;99:e20051.

22. Jaskolka JD, Asch MR, Kachura JR, et al. Needle tract seeding after radiofrequency ablation of hepatic tumors. J Vasc Interv Radiol. 2005;16:485-91.

23. Cabibbo G, Enea M, Attanasio M, et al. A meta-analysis of survival rates of untreated patients in randomized clinical trials of hepatocellular carcinoma. Hepatology. 2010;51:1274-83.

24. Bruix J, Raoul JL, Sherman M, et al. Efficacy and safety of sorafenib in patients with advanced hepatocellular carcinoma: subanalyses of a phase III trial. J Hepatol. 2012;57:821-9.

25. Sakamoto K, Nakashima K, Migo S, et al. A study of treatment for extrahepatic metastasis after sugical treatment of hepatocellular carcinoma. Jpn J Gastroenterol Surg. 2002;35:116-9.

26. Nakayama H, Takayama $T$, Makuuchi M, et al. Resection of peritoneal metastases from hepatocellular carcinoma-PubMed. Hepatogastroenterology. 1999;46:1049-52.

27. Lin CC, Liang HP, Lee HS, et al. Clinical manifestations and survival of hepatocellular carcinoma patients with peritoneal metastasis. J Gastroenterol Hepatol. 2009;24:815-20.

28. Takemura N, Hasegawa K, Aoki T, et al. Surgical resection of peritoneal or thoracoabdominal wall implants from hepatocellular carcinoma. Br J Surg. 2014;101:1017-22.

29. Llovet JM, Ricci S, Mazzaferro V, et al. Sorafenib in advanced hepatocellular carcinoma. N Engl J Med. 2008;359:378-90.

30. Kanayama M, Nagai H, Sumino Y. Influence of the etiology of liver cirrhosis on the response to combined intra-arterial chemotherapy in patients with advanced hepatocellular carcinoma. Cancer Chemother Pharmacol. 2009;64:109-14.

31. Ueshima K, Kudo M, Takita M, et al. Hepatic arterial infusion chemotherapy using low-dose 5-fluorouracil and cisplatin for advanced hepatocellular carcinoma. Oncology. 2010;78:148-53. 
32. Obi S, Yoshida H, Toune R, et al. Combination therapy of intraarterial 5-fluorouracil and systemic interferon-alpha for advanced hepatocellular carcinoma with portal venous invasion. Cancer. 2006;106:1990-7.

33. Furuse J, Okusaka T, Kaneko S, et al. Phase I/II study of the pharmacokinetics, safety and efficacy of S-1 in patients with advanced hepatocellular carcinoma. Cancer Sci. 2010;101:2606-11.

34. Kudo M, Moriguchi M, Numata K, et al. S-1 versus placebo in patients with sorafenib-refractory advanced hepatocellular carcinoma (S-CUBE): a randomised, double-blind, multicentre, phase 3 trial. Lancet Gastroenterol Hepatol. 2017;2:407-17.

35. Ye L, Liang R, Zhang J, et al. Postoperative albumin-bilirubin grade and albumin-bilirubin change predict the outcomes of hepatocellular carcinoma after hepatectomy. Ann Transl Med. 2019;7:367-367.

36. $\mathrm{Xu} \mathrm{L}, \mathrm{Wu}, \mathrm{Ju} \mathrm{W}$, et al. Application of the albumin-bilirubin grade in predicting the prognosis of patients with hepatocellular carcinoma: a systematic review and meta-analysis. Transpl Proc. 2019;51:3338-46.

37. Oh IS, Sinn DH, Kang TW, et al. Liver function assessment using albumin-bilirubin grade for patients with very early-stage hepatocellular carcinoma treated with radiofrequency ablation. Dig Dis Sci. 2017;62:3235-42.

38. Gui B, Weiner AA, Nosher J, et al. Assessment of the AlbuminBilirubin (ALBI) Grade as a Prognostic Indicator for Hepatocellular Carcinoma Patients Treated with Radioembolization. Am J Clin Oncol Cancer Clin Trials. 2018;41:861-6.

39. Su T-SS, Yang H-MM, Zhou Y, et al. Albumin-Bilirubin (ALBI) versus Child-Turcotte-Pugh (CTP) in prognosis of HCC after stereotactic body radiation therapy. Radiat Oncol. 2019;14:50.

40. Kuo $\mathrm{YH}$, Wang JH, Hung $\mathrm{CH}$, et al. Albumin-Bilirubin grade predicts prognosis of HCC patients with sorafenib use. J Gastroenterol Hepatol. 2017;32:1975-81.

41. Hiraoka A, Kumada T, Atsukawa M, et al. Important clinical factors in sequential therapy including lenvatinib against unresectable hepatocellular carcinoma. Oncology. 2019;97:277-85.
42. Ueshima K, Nishida N, Hagiwara S, et al. Impact of baseline ALBI grade on the outcomes of hepatocellular carcinoma patients treated with lenvatinib: a multicenter study. Cancers (Basel). $2019 ; 11$

43. Hatanaka T, Naganuma A, Shibasaki M, et al. The role of the albumin-bilirubin score for predicting the outcomes in Japanese patients with advanced hepatocellular carcinoma treated with ramucirumab: a real-world study. Oncology. 2020.

44. Zhu AX, Kang YK, Yen CJ, et al. Ramucirumab after sorafenib in patients with advanced hepatocellular carcinoma and increased $\alpha$-fetoprotein concentrations (REACH-2): a randomised, double-blind, placebo-controlled, phase 3 trial. Lancet Oncol. 2019;20:282-96.

45. Wada Y, Takami Y, Ryu T, et al. A case of advanced hepatocellular carcinoma with partial response after continuous ramucirumab treatment beyond radiological progression. Case Rep Oncol. 2020;13:379-84.

46. Kudo M, Galle PR, Llovet JM, et al. Ramucirumab in elderly patients with hepatocellular carcinoma and elevated alphafetoprotein after sorafenib in REACH and REACH-2. Liver Int. 2020;40:2008-20.

47. Wang D, Yang X, Lin J, et al. Comparing the efficacy and safety of second-line therapies for advanced hepatocellular carcinoma: a network meta-analysis of phase III trials. Therap Adv Gastroenterol. 2020;13.

Publisher's Note Springer Nature remains neutral with regard to jurisdictional claims in published maps and institutional affiliations. 